\title{
MOBILE APPLICATIONS FOR HEALTH CARE SUPPORT
}

\author{
Dejan Zillidejan.zilli@nova.vizija.si, Jožica Leskovšek jozica.leskovsek@nova.vizija.si \\ Nova Vizija, Information Engineering and Consulting, Celjska 9, 3310 Žalec, Slovenia \\ Srečko Natek srecko.natek@mfdps.si \\ Nova Vizija, Information Engineering and Consulting, Celjska 9, 3310 Žalec, Slovenia \\ International School for Social and Business Studies, Mariborska 7, 3000 Celje, Slovenia \\ Dušan Lesjak, dusan.lesjak@fm-kp.si \\ University of Primorska, Faculty of Management, Cankarjeva 5, 6000 Koper, Slovenia \\ International School for Social and Business Studies, Mariborska 7, 3000 Celje, Slovenia
}

\begin{abstract}
Home health care consists of different areas; mainly medical home care, house calls and emergency medical assistance. Doctors, nurses and other health care professionals need patient information, but are often unable to access the information technology (IT) support that is normally used at their workplace. Health care information systems are usually not mobile, because they have to meet high security and privacy requirements for patient personal and medical data. During the research, a pilot mobile healthcare solution for house calls, integrated with health care institution's information system, was developed. Several Slovenian health center and doctors participated in the research, sharing key healthcare and mobile IT solutions knowledge. Application of research results and mobile solutions in other areas of health care, integration of solutions with the national health care information system, and integration of solutions with other healthcare information systems are challenges for further research.
\end{abstract}

Keywords. Home Health Care, House Call, Health Care, Mobile Application, Mobile Solution, Security, Privacy

\section{INTRODUCTION}

Home health care [12] is the oldest form of patient treatment. In a not-so-distant past, medical practitioners mainly attended to patients at their homes. Today, doctors mainly carry out their work in clinics, where they have all the necessary support from various professionals, facilities, and of course the information system with patient data. Home health care includes different areas: home visits, emergency medical assistance and medical home care [17].

In Slovenia, as elsewhere, home health care is well organized and performed by so-called family or on-call doctors and mainly deals with care for patients with chronic illnesses or acute diseases, and help for the elderly, disabled or dying patients. The citizens are overall satisfied by the health care system on a primary level.

The number of home visits is declining compared with the number of visits in a clinic [18]. The doctors responsible for house calls could benefit from access to medical records that are completed by all who participate in the treatment of various illnesses and/or injuries of a patient. However, there is a problem with the availability of these records during a home visit.

Nova Vizija, a software firm, conducted research in 2014 in collaboration with 5 doctors from two health centers, which concluded that health care IT solutions can substantially help doctors who work in the field. During a home visit doctors are usually almost completely isolated from the IT support, which is otherwise available at their workplace in health centers. Unfortunately, existing Slovenian health care IT solutions are not mobile although the mobile infrastructure in Slovenia is good, providing adequate mobile services also for health care mobile applications. 
The research focused on house calls carried out by general practitioners but also included specialists from other health care disciplines $[11,12]$.

The research investigated mobile IT solutions for home health care that would give doctors access to patient medical records, while solving the problems of privacy of such sensitive medical data [15]. For this reason, the research focused on cloud service as a solution for safe communication between health care institution's information system and doctor's mobile device, using personal digital certificate to satisfy the most demanding security and privacy requirements.

\section{RESEARCH}

\section{Mobile IT Solution Design}

During the mobile application design, the focus was on three main features: security and privacy, ease of use, and portability. Security and privacy are often the main concerns when sharing healthcare data. Ease of use is very important as doctors, like all users of IT solutions, usually only benefit from applications with an excellent design. Portability is the main benefit of mobile applications, especially useful for fieldwork.

The key research question was about data security and privacy: What level of data security is essential and possible for mobile healthcare applications. Studies in this field advocate that privacy and security of personal health information are very important for future healthcare solutions development [6]. Furthermore, due to the possibility of serious threats on mobile devices, mobile health application security is even more important [2].

The key requirements for solution design were:

- High level of data security,

- Support for different mobile phone and tablet operating systems (at least Android and iOS),

- $\quad$ Support for off-line usage,

- Display of key patient medical information required by the doctor,

- Highly transparent display of medical data,

- Simple and efficient interface.

These requirements led to the development of an application, which enables transformation, transmission and display of relevant data from the institution's medical database to a mobile device exploiting the potential of semantic computing management [3].

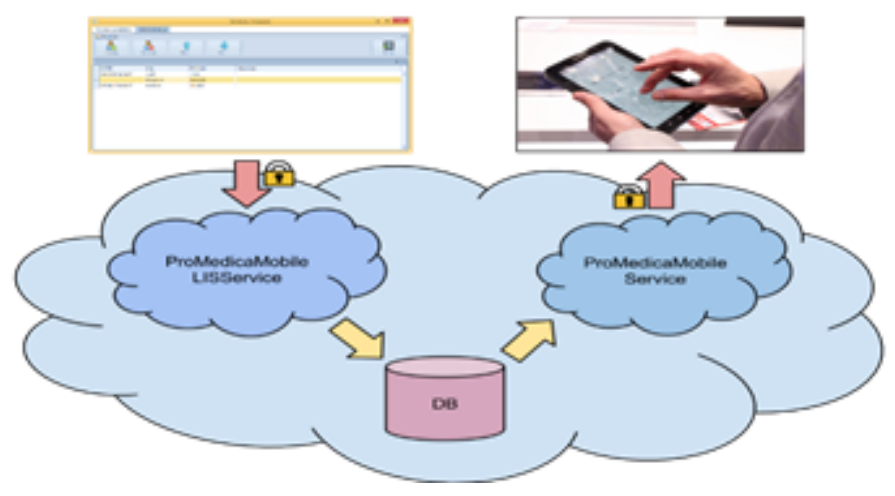

Figure 1. Architecture of the Mobile IT Solution for Home Health Care 


\section{Data Model and User Interface Design}

Two health centers and five family doctors in Slovenia, coordinated by the Nova Vizija research team, were involved in the research project and mainly contributed the health care domain knowledge e.g. medical data, processes and documentation.

The medical data are the foundation of doctor - patient communication [8]. The medical data that can be available on doctor's mobile device include: patient's personal data, reason for the house call, basic information on chronic diseases, permanent therapy, prescribed medications in the last period, allergies (including allergies to medications), last lab reports, any issued orders for medical accessories, information about the last few visits to the clinic (anamneses, statuses, diagnoses, prescribed medicines...).

Following the initial design of the data model and user interface, the team of family doctors evaluated the application's form (user interface), features and overall application design.

\section{Patient Medical Record Transmission}

Before a data package is transmitted to a mobile device, a nurse enters a reason for the house call (anamnesis vitae) and determines the schedule of house calls (i.e. the order in which the doctor should visit the patients).

During the medical data package preparation, doctor's professional authorization card is also presented and the data from the Health Insurance Institute's on-line system is transmitted as well as any prescribed medicines and open orders for medical accessories.

The mobile IT solution combines all patient medical data into a package and sends it to the cloud where it is stored temporarily and from where it is downloaded onto a mobile device. Such a design, illustrated in Figure 1, allows for the data to be prepared before the doctor leaves the clinic, as well as afterwards. The only requirement for medical information to be downloaded is that the mobile device is connected to the Internet at any time during the doctor's trip to the patient's home. Modern technology is used for cloud services and communication with the cloud (Windows Communication Foundation - WCF / Simple Object Access Protocol - SOAP, Secure Sockets Layer SSL).

\section{Data Access and Data Security}

During the design and development of the solution, special consideration was given to data security and privacy, as patient medical data falls into the category of particularly sensitive personal information, as discussed by several authors $[2,4,6]$.

Solution design included data access limitations for different types of users. For example, nurses, who transmit the data for the doctor's house call, do not see any medical data [13].

Before it is sent to the cloud, the data package is encrypted with the public key of the designated doctor's digital certificate and can therefore not be accessed by anyone else. The encrypted data package is sent to the cloud where it is temporarily stored. The doctor's private key is not transmitted through the cloud at any point, so even the cloud administrators are not able to decrypt the data. The data package is encrypted with a random key and a symmetric AES-256 algorithm, while RSA5 algorithm and a private key X.5096 of the digital certificate are used for encoding the random key.

Only the designated (authorized) doctor can download the data package from the cloud onto their mobile device. Once the medical data has been downloaded onto the mobile device, the data package is erased from the cloud and thus no longer available. The connection between the cloud and the mobile device is protected by using the HTTPS protocol. Encrypted data are stored on a mobile device only temporarily, for 24 hours or according to the selected settings. 
Using the mobile solution on a mobile device, the doctor decrypts the data with the private key of the digital certificate. To ensure additional security, the private key on the device is encrypted with a personal user PIN (AES256), which has to be entered when the doctor signs in (a 4- to 6-digit code set by the doctor, which can be changed at any time).

The data can therefore be accessed and reviewed only by the doctor for whom the data package was prepared and only on mobile devices that had the private key of the digital certificate installed in the mobile solution installation process. Data security level is in line with the recommendations of the Slovenian Information Commissioner [19] for health care IT solutions, which was confirmed by the Information Commissioner prior to the commencement of the mobile solution development.

\section{RESULTS - MOBILE IT SOLUTION}

The design of the mobile solution focused on three main features: security - privacy, ease of use and portability.

Security - privacy was ensured through sound planning and the use of proven and reliable encryption technologies, which nowadays represent the standards in data protection provision.

Ease of use was achieved through the adoption of modern standards in mobile user interface design, whereby the application is adapted to the users who in turn are not required to adjust to the application, as is often the case in practice (intuitive PIN input, sliding navigation screens, etc.). Screenshots of the application in Figure 2 are examples how this principles can be implemented during user interface design.

Application portability was ensured through the use of the latest frameworks for the mobile application design (ionicframework, AngularJS, Cordova, HTML5, JavaScript). The result is a hybrid HTML5 app that adapts to the size of the screen and can be used on any type of mobile devices (phones, tablets) and on any platform (Android, iOS).
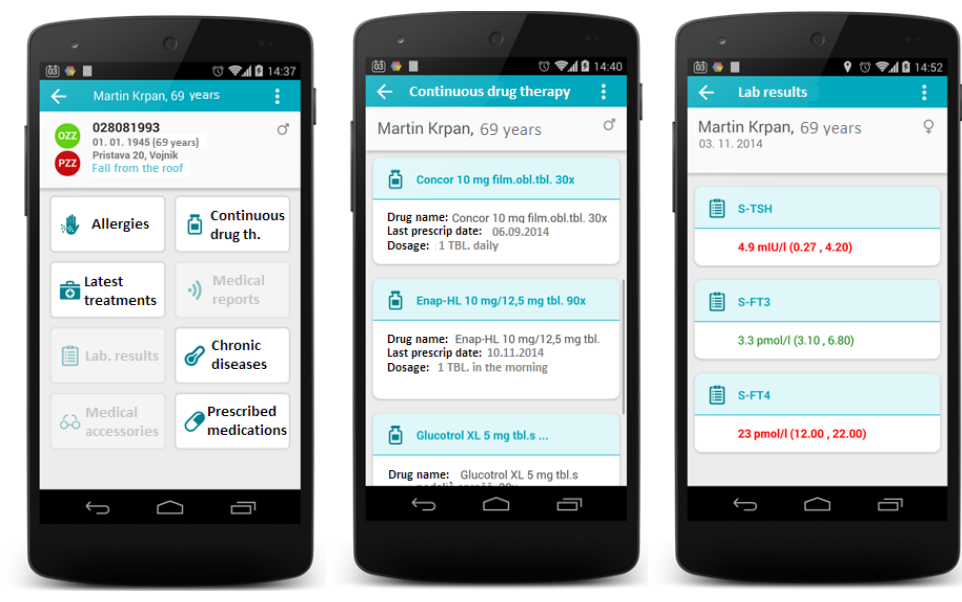

Figure 2. Example Screenshots of the App

All available technology and other measures were identified, tested, evaluated and implemented in the pilot mobile solution.

The research took about a year with a research resource of one man per year. The main challenges were the data fields sequence in the user interface and privacy issues.

The technology and features incorporated in the solution define the essential data security level and prove that it is possible to meet the high data-security requirements. 
Following the testing of over twenty scenarios in a two-month period, the evaluation of the pilot mobile solution established that mobile data availability presents a real benefit, but that there are some weakness with regards to the data field sequence in the user interface. The evaluation finally proved that the doctors are better informed and thus able to provide better health care. Doctors also benefit by saving the time spent on individually gathering patient data in health care centers.

In addition, the research recognized that healthcare professionals have a positive attitude towards mobile IT, as also proved by other research [7,14]. The application is very user friendly with highly automated data transfer and updates. However there are still some issues using cloud services as patient data are available to the certified user for a certain period of time (e.g. two days). So decision between maximum privacy and functionality has to be accepted in near future.

\section{FURTHER DEVELOPMENT}

After a successful implementation of the mobile solution, the solution could be expanded to support other health teams who work in the field. For example, treatment at home is often intertwined and complementary to health care, which is performed by the home care nurse. In addition, sports medicine doctors who go abroad with their clients to help them prepare for competitions could make a good use of mobile IT applications and sportsman electronic medical records, as well as doctors performing urgent medical assistance who often do not have access to the data on patients, etc.

The main challenge of a further development of the solution from a technical point of view is its integration with the national health care information system (interoperable backbone) using the openEHR standard. This would give doctors access to complete patient medical data and would no longer limit them to information that is available in their institution.

Once the data on how users use the solution is collected, it will be possible to transform the solution into a sustainable mobile health solution, as described by Sultan and Mohan [16].

Integration with other providers of health care information systems is also planned. The use of service-oriented architecture (SOA) makes the service ready to be used with information systems from other providers. Their customers could use the mobile app, providing that the providers adapt their information systems to allow for the encrypted patient medical data packages to be prepared and sent to the cloud. In order to facilitate the integration of providers, it would be sensible to prepare a library of installation files for mobile devices and to consider any other possibility of installing applications and user administration (e.g. Web interface).

The cooperation with other software providers could be even stronger. Doctors could have the ability to request medical information from the patient's personal physician using their mobile device and have it automatically forwarded by the back end information system. This idea is still in its early stages due to the need to explore in detail the safety aspects of data transmission on request.

\section{CONCLUSIONS}

Mobile IT solutions for home health care support will play an important role in the future, primarily for patients who would like the doctors who visit them to have access to the data on their health conditions, diseases, allergies, medicament therapy, etc. Mobile IT solutions exceed the rigid limitations of the otherwise-powerful concept of a family doctor whose main advantages are data and knowledge of patients. New possibilities offered by mobile IT solutions will support the society to build a better field health care service.

Application of research results and mobile solutions in other areas of health care, integration of the application with the national health care information system, and integration of the application with other providers of healthcare information systems are challenges for further research. 


\section{REFERENCES}

1. Angularjs, https://angularjs.org

2. Bajwa, M., (2014). Mobile Health Security: Threats and Mitigation, The College of St. Scholastica.

3. Bellini, P., Bruno, I., Cenni, D., Fuzier, A., Nesi, P. and Paolucci, M., (2012). Mobile Medicine: semantic computing management for health care applications on desktop and mobile devices. Multimedia Tools and Applications, 58 (1), 41-79.

4. Chaudhary, R. and Ward, J.J., (2014). A Practical Approach to Health Care Information Security. Journal of Health Care Compliance, 16(3), 16,66.

5. Cordova Apache, http://cordova.apache.org

6. Dimovski, B. (2014). Mobile Phones in Healthcare: Privacy and Security of Personal Health Information (PHI), The College of St. Scholastica.

7. Gund, A., Lindecrantz, K., Schaufelberger, M., Patel, H. and Sjoqvist, B.A. (2012). Attitudes among healthcare professionals towards ICT and home follow-up in chronic heart failure care. BMC Medical Informatics and Decision Making, 12, 138.

8. Haluza, D. and Jungwirth, D. (2014). ICT and the future of health care: Aspects of doctor - patient communication. International Journal of Technology Assessment in Health Care 30 (3), 298-305.

9. HTML5, http://www.w3.org/TR/html5/

10. Ionicframework, http://ionicframework.com

11. Mouttham, A., Kuziemsky, C., Langayan, D., Peyton, L., Pereira, J. (2011). Interoperable support for collaborative, mobile and accessible health care. Information Systems Frontiers, Springer, 14: 73-85.

12. Parkinson, C.I. (2012). Primary care physicians' perceptions of the house call for chronic conditions as it relates to quality health care in the patient population 65 years of age and older, $D^{\prime}$ Youville College.

13. Perez, B., Diez, I., Coronado, M. (2015). Privacy and Security in Mobile Health Apps: A Review and Recommendations. Journal of Medica Systems, Springer, 39: 181.

14. Quanbeck, A.R., Gustafson, D.H., Marsch, L.A., Mctavish, F., Brown, R.T., Mares, M., Johnson, R., Glass, J.E., Atwood, A.K. and McDowell, H. (2014). Integrating addiction treatment into primary care using mobile health technology: protocol for an implementation research study. Implementation Science, 9, 65.

15. Silva, B., Rodrigues, J., Canelo, F., Lopes, I., Lloret, J. (2014). Towards a cooperative security system for mobile-health applications. Electronic Commerce Research, Springer.

16. Sultan, S. and Mohan, P. (2013). Transforming usage data into a sustainable mobile health solution. Electronic Markets, 23 (1), 63-72.

17. Thume, E., Facchini, L.A., Wyshak, G., and Campbell, P. (2011). The Utilization of Home Care by the Elderly in Brazil's Primary Health Care System. American Journal of Public Health, 101(5), 868-74.

18. Zivcec-Kalan G, Kersnik J, Zonik L. (2004). Obremenjenost, preobremenjenost in zadovoljstvo z delom pri zdravnikih družinske medicine. [Workload burden, overload and satisfaction with work among family doctors] In: Kersnik J (ed.). Ulkusna bolezen, hipertenzija, zaščitni dejavniki zdravil na mikrocirkulacijo, KOPB, astma, možganska kap: zbornik predavanj, (Zbirka PiP). [Ulcer disease, hypertension, protective factors of microcirculation drugs, COPD, asthma, stroke: essays (PiP Collection)] Ljubljana: Slovenian Medical Association, Slovenian Family Medicine Society, 100. http://www.drmed-mb.org/wpcontent/uploads/2010/11/\%C4\%8Clanki-iz-zbornikov-Janko-Kersnik.pdf (10. 4. 2015)

19. Informacijski pooblaščenec. Smernice in priročniki. [Information Commissioner. Guidelines and manuals] https://www.ip-rs.si/publikacije/prirocniki-in-smernice/ (17. 4. 2015) 